\title{
Effects Of Higher Order Chemical Reactions and Slip Boundary Conditions on Nanofluid Flow
}

\author{
D. Rajani ${ }^{1}$, K. Hemalatha ${ }^{2}$, M.V.D.N.S.Madhavi ${ }^{3}$ \\ 1,2,3 Department of Mathematics, V.R.Siddhartha Engineering College, Vijayawada - 520007, India \\ Research Scholar, Krishna University, Machilipatnam - 521001, India
}

\begin{abstract}
We investigate the effects of higher order chemical reactions on coupled heat and mass transfer from a permeable stretching sheet in nanofluid flow with slip boundary conditions. Nanofluids play an important role in emerging nanotechnology and biotechnology industries where they find application in heat transfer processes. We study the effects of the order of the chemical reaction and chemical reaction parameter. The highly nonlinear and coupled governing differential equations were solved numerically. The solution depends on Brownian motion number, thermophoresis number, thermal convective parameter, mass convective parameter and double diffusive buoyancy parameter. The effects of various engineering parameters are investigated.
\end{abstract}

Keywords: Nanofluid, Stretching sheet, Chemical reaction, Slip boundary condition.

\section{INTRODUCTION}

The flow and heat transfer over a stretching surface is applicable in many engineering processes such as in the aerodynamic extrusion of plastic sheets, metallurgy, the cooling of metallic plates in a cooling bath, polymer extrusion, textile and glass fiber production. The concept of stretching sheet was first used by Crane [1] to study the flow of an incompressible viscous fluid with a linearly varying surface velocity. Later, several authors extended this work to various aspects of fluid flow and heat transfer over a stretching sheet (Erickson et al. [2] Grubka and Bobba [3], Chen [4], Abo-Eldahab and Abd El-Aziz [5]).

The no-slip boundary condition at the interface between a solid surface and a liquid or gas applies in commonly occurring situations. However, there are cases when this condition is not appropriate, for instance, the no slip boundary condition is inadequate in many situations such as in gas flow in microfluidic and nanofluidic devices $([6,7,8,9,10])$. The slip boundary conditions are encountered in the modeling of turbulent fluid flows. The numerical solution of fluid flow equations with slip boundary conditions is important for both modeling and optimization purposes. A survey of literature indicates that much attention has been given to slip effect, especially in polymer industry (polymer melts), which exhibit a macroscopic wall slip. Wang [11] found the closed form similarity solution of the Navier-Stokes equations for flow due to a stretching sheet with partial slip. The effects of slip at the boundary for the flow of a viscous incompressible fluid past a stretching sheet were studied by Andersson [12]. Slip effects on boundary layer stagnation-point flow and heat transfer from a shrinking sheet were studied by Bhattacharyya et al. [13]. They found dual solutions of the governing equations and showed that the velocity and thermal boundary layer thicknesses in the first solution are always smaller than that of the second solution. Fang et al. [14] studied the viscous flow over a shrinking sheet with a second order slip flow model. Bhattacharyya and Layek [15] studied the flow solution of MHD boundary layer flow with diffusion and chemical reaction over a porous flat plate with suction/blowing.

The radiation effect is important in space technology and high temperature processes. Hossain and Takhar [16], Takhar et al. [17], Hossain et al. [18] investigated the effect of radiation on heat transfer problems in detail. Mukhopadhyay and Gorla [19] studied the effects of partial slip on boundary layer flow past a permeable exponential stretching sheet with thermal radiation. Chemical reactions may be classified as either homogenous or heterogeneous chemical reactions. In homogeneous reactions all the reactants are in the same phase while in heterogeneous reactions the reactants are in two or more phases. In most cases, the reaction rate depends on the concentration of the species itself. A reaction is said to be of order $n$ if the rate of reaction is directly proportional to the $n$th power of species concentration. Chemical reaction plays an important role in chemical industries, agriculture, food processing and polymer production. Rahman and AlLawatia [20] studied the effects of higher order chemical reactions on micropolar fluid flow on a power law permeable stretched sheet with variable concentration in a porous medium. Their results indicate that for higher order reactions the rate of increase in mass transfer is less compared to the rate 
of increase for lower order reactions. The mass transfer rate increases significantly with an increase in the chemical reaction parameter $\beta$. The diffusion of chemical reactive species with first-order and higherorder reactions over a linearly stretching sheet was studied by Anderson et al. [21]. Takhar et al. [22] investigated the flow and mass diffusion of a chemical species with first-order and higher-order reactions over a continuously stretching sheet.

Nanofluids possess unique thermal properties that are different from those of conventional heat transfer fluids. These properties include significantly enhanced heat transfer coefficients and strong temperature dependent thermal conductivity. The boundary layer flow and heat transfer in nanofluids over a stretching sheet has applications over a broad spectrum of science and engineering disciplines. Ibrahim and Shankar [24] studied the MHD boundary layer flow and heat transfer in a nanofluid flow past a permeable stretching sheet with slip boundary conditions. They found that the skin friction coefficient decreases with an increase in the velocity slip parameter. Partial slip flow and heat transfer over a stretching sheet in a nanofluid was studied by Sharma et al. [25]. They observed that the velocity slip parameter reduced the

$$
T_{w}(x)=T_{\infty}+A_{1}\left(\frac{x}{l}\right)^{2}, C_{w}(x)=C_{\infty}+A_{2}\left(\frac{x}{l}\right)^{2}, \phi_{w}
$$
Temperature, concentration and nanoparticle volume fraction of the ambient fluid. In the present paper we revisit the problem of Ibrahim \& Shankar [24] and studied the effects of thermal convective, mass convective, double diffusive buoyancy parameters and order of chemical reaction parameters. The nanoparticle with spherical in shape and the particle size is uniform. So the viscosity of the fluid only depends on the volume heat transfer rate.

The present study analyzes the effects of the chemical reaction parameter on velocity, temperature, solute concentration, nanoparticle concentration, heat transfer, mass transfer and nanoparticle mass transfer for different orders of chemical reactions. We have investigated the thermal convective parameter and nanoparticle mass convective parameter in the momentum equation. The radiation and chemical reaction effects are considered in the energy and solute concentration equations. The boundary conditions used in the study are the velocity slip, thermal slip, solute slip and nanoparticle slip conditions.

\section{MATHEMATICAL ANALYSIS}

We consider a two dimensional steady laminar incompressible boundary layer nanofluid flow over a stretching sheet in a porous medium. The problem is described in a rectangular coordinate system with the origin of the system located at the slit where the sheet is drawn. The coordinate is chosen such that the $x$ axis is along the sheet and the $y$-axis is normal to the sheet is shown in Fig 1. The temperature, concentration, nanoparticle volume fraction at the surface is

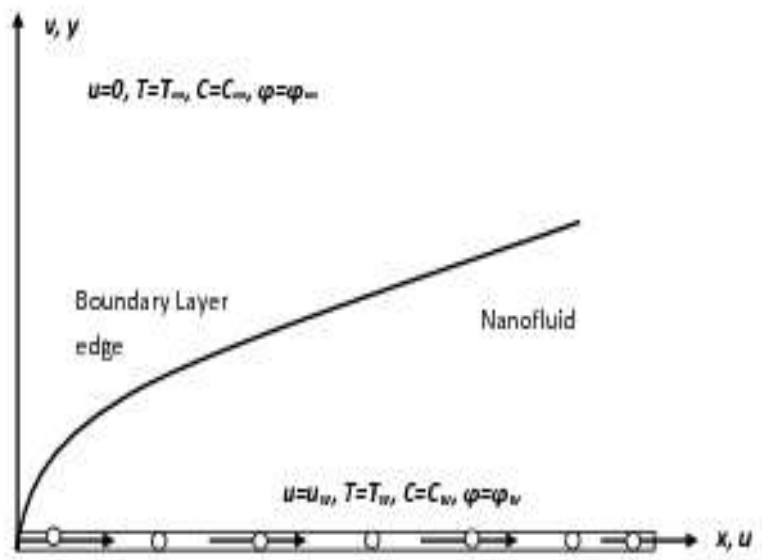

Figure 1: Physical model and coordinate system 


$$
\begin{array}{ll} 
& \frac{\partial u}{\partial x}+\frac{\partial v}{\partial y}=0, \\
& u \frac{\partial u}{\partial x}+v \frac{\partial u}{\partial y}=v \frac{\partial^{2} u}{\partial y^{2}} \pm \frac{\left(1-\phi_{\infty}\right) K g \rho f_{\infty}}{\mu}\left(\beta_{T} \frac{\partial T}{\partial y}+\beta_{c} \frac{\partial C}{\partial y}\right) \mp \frac{\left(\rho_{p}-\rho f_{\infty}\right) K g}{\mu} \frac{\partial \phi}{\partial y}, \\
& u \frac{\partial T}{\partial x}+v \frac{\partial T}{\partial y}=\alpha_{m} \frac{\partial^{2} T}{\partial y^{2}}+\tau\left\{D_{B} \frac{\partial T}{\partial y} \frac{\partial \phi}{\partial y}+\frac{D_{T}}{T_{\infty}}\left(\frac{\partial T}{\partial y}\right)^{2}\right\}-\frac{1}{\rho f_{\infty} C_{p}} \frac{\partial q_{r}}{\partial y}, \\
& \frac{1}{\varepsilon}\left(u \frac{\partial C}{\partial x}+v \frac{\partial C}{\partial y}\right)=D_{s} \frac{\partial^{2} C}{\partial y^{2}}+D_{C T} \frac{\partial^{2} T}{\partial y^{2}}-K_{0}\left(C-C_{\infty}\right)^{n}, \\
& \frac{1}{\varepsilon}\left(u \frac{\partial \phi}{\partial x}+v \frac{\partial \phi}{\partial y}\right)=D_{B} \frac{\partial^{2} \phi}{\partial y^{2}}+\frac{D_{T}}{T_{\infty}} \frac{\partial^{2} T}{\partial y^{2}} \\
\text { where } \quad \alpha_{m}=\frac{k_{m}}{(\rho c)_{f}} \text { and } \tau=\frac{\varepsilon(\rho)_{p}}{(\rho c)_{f}}
\end{array}
$$

The boundary conditions for equations (1)-(5) are

$$
\begin{aligned}
u & =u_{w}+L \frac{\partial u}{\partial y}, v=0, \\
T & =T_{w}+K_{1} \frac{\partial T}{\partial y}, C=C_{w}+K_{2} \frac{\partial T}{\partial y}, \\
\phi & =\phi_{w}+K_{3} \frac{\partial \phi}{\partial y} \text { at } y=0, \\
u & \rightarrow 0, T \rightarrow T_{\infty}, C \rightarrow C_{\infty}, \phi \rightarrow \phi_{\infty} \text { as } y \rightarrow \infty
\end{aligned}
$$

where $u_{w}=b x, L, K_{1}, K_{2}$ and $K_{3}$ are the velocity, velocity slip, thermal slip, solute slip and nanoparticle volume fraction slip factors respectively. When $L=$ $K_{1}=K_{2}=K_{3}=0$, the no - slip condition is recovered, $l$ is the reference length of the stretching sheet. The above conditions are valid when $x \ll l$. Furthermore $u$ and $v$ are the velocity components in the $x$ and $y$ directions respectively $v$ is the kinematic viscosity, $K$ is the permeability of porous medium, $g$ is the acceleration due to gravity, $\rho f_{\infty}$ is the density of the base fluid, $\mu$ is the viscosity of the of the nanofluid, $\beta_{T}$ is the thermal volumetric expansion coefficient of the nanofluid, $T$ is the temperature, $\beta_{C}$ is the solute volumetric expansion coefficient of the nanofluid, $C$ is the concentration, $\rho_{p}$ is the density of nanoparticle, $\phi$ is the nanoparticle concentration, \pm sign represent the buoyancy assisting and opposing flow regions, $\alpha_{m}$

$q_{r}=-\frac{4 \sigma^{*}}{3 k^{*}} \frac{\partial T^{4}}{\partial y}$, is the thermal diffusivity of the base fluid, $\tau$ is ratio of the effective heat capacity of the nanoparticle material and the heat capacity of the fluid, $D_{B}$ is the Brownian diffusion coefficient, $D_{T}$ is the thermophoretic diffusion coefficient, $C p$ is the specific heat at constant pressure, $q_{r}$ is the radiative heat flux, $\varepsilon$ is the porosity of the porous medium, $D s$ is the solute diffusivity, $D_{C T}$ is the Soret type diffusivity, $K_{0}$ is the chemical reaction parameter, $n$ is the order of the chemical reaction, $k_{m}$ is the thermal conductivity, $(\rho c)_{f}$ is the heat capacity of the base fluid and $(\rho c)_{p}$ is the effective heat capacity of the nanoparticle material.

Following Rosseland's approximation, the radiative heat flux $q_{r}$ is modeled from Modest [26] as

where $\sigma *$ is the Stefan-Boltzman constant, $k^{*}$ is the mean absorption coefficient. Assuming the temperature differences within the flow are sufficiently small such that $T^{4}$ may be expressed as a linear function of $T^{4} \cong$ $4 T_{\infty}^{3} T-3 T_{\infty}^{4}$,we have

$\frac{\partial q_{r}}{\partial y}=-\frac{16 \sigma^{*} T_{\infty}^{3}}{3 k^{*}} \frac{\partial^{2} T}{\partial y^{2}}$.

\section{TRANSFORMATION OF EQUATIONS}

The continuity equation (1) is satisfied by introducing a stream function $\psi(x, y)$ such that $u=\frac{\partial \psi}{\partial y}$ and $=-\frac{\partial \psi}{\partial x}$,

where $\psi=(b v)^{\frac{1}{2}} x f(\eta), f(\eta)$ is the dimensionless stream function and $\eta=(b / v)^{\frac{1}{2}} y$.

The velocity components are given by

$u=b x f^{\prime}(\eta)$ and $=-(b v)^{\frac{1}{2}} f(\eta)$.

The temperature, solute concentration and nanoparticle concentration are represented as

$T=T_{\infty}+\Delta T \theta(\eta)$

$C=C_{\infty}+\Delta C S(\eta)$

$\phi=\phi_{\infty}+\Delta \phi \gamma(\eta)$, 
where $\theta(\eta), S(\eta)$ and $\gamma(\eta)$ are the dimensionless temperature, dimensionless solute concentration and dimensionless nanoparticle concentration, $\Delta \mathrm{T}=$ $\mathrm{T}_{\mathrm{w}}-\mathrm{T}_{\infty}, \Delta \mathrm{C}=\mathrm{C}_{\mathrm{w}}-\mathrm{C}_{\infty}, \Delta \phi=\phi_{\mathrm{w}}-\phi_{\infty}$. On using $f^{\prime \prime \prime}+f f^{\prime \prime}-f^{\prime 2} \pm \lambda_{1}\left(\theta^{\prime}+N c S^{\prime}\right) \mp \lambda_{2} \gamma^{\prime}=0$,

$\left(1+\frac{4}{3} R\right) \theta^{\prime \prime}+\operatorname{Prf} \theta^{\prime}-2 \operatorname{Pr} f^{\prime} \theta+\operatorname{PrNb} \theta^{\prime} \gamma^{\prime}+\operatorname{PrNt} \theta^{2}=0$,

$S^{\prime \prime}+S c f S^{\prime}-2 S c f^{\prime} S+S c S r \theta^{\prime \prime}-S c \beta S^{n}=0$,

$\gamma^{\prime \prime}+L e f \gamma^{\prime}-2 L e f^{\prime} \gamma+\frac{N t}{N b} \theta^{\prime \prime}=0$.

equations (9) and (10), equations (2) - (6) transform into the following two-point boundary value problem

The boundary conditions are

$f(0)=0, f^{\prime}(0)=1+G_{1} f^{\prime \prime}(0), f^{\prime}(\infty) \rightarrow 0$,

$\theta(0)=1+G_{2} \theta^{\prime}(0), \theta(\infty) \rightarrow 0$,

$S(0)=1+G_{3} S^{\prime}(0), S(\infty) \rightarrow 0$,

$\gamma(0)=1+G_{4} \gamma^{\prime}(0), \gamma(\infty) \rightarrow 0$,

where the primes denote differentiation with respect to $\eta$. The non-dimensional constants in equations (11)- (18) are thermal convective parameter $\lambda_{1}$, nanoparticle mass convective parameter $\lambda_{2}$, double diffusive buoyancy parameter $N c$, Radiation parameter $R$, Prandtl number $P r$, Brownian motion parameter $N b$, thermophoresis

parameter $N t$, Schmidt number $S c$, Soret number $S r$, non-dimensional chemical reaction parameter $\beta$, Lewis number $L e$, velocity slip parameter $G_{1}$, thermal slip parameter $G_{2}$, solutal slip parameter $G_{3}$ and nanoparticle slip parameter $G_{4}$. These parameters are defined as

$$
\begin{aligned}
& \lambda_{1}=\frac{G r_{x}}{R_{x}^{\frac{3}{2}}}, \lambda_{2}=\frac{G n_{x}}{R e_{x}^{\frac{3}{2}}}, G r_{x}=\frac{\left(1-\phi_{\infty}\right) K g \beta_{T} \rho f_{\infty} \Delta T x^{2}}{\mu v^{2}}, R e_{x}=\frac{x u_{w}}{v}, G n_{x}=\frac{\left(\rho_{p}-\rho f_{\infty}\right) K g \Delta \phi x^{2}}{\mu v^{2}}, N c=\frac{\beta_{C} \Delta C}{\beta_{T} \Delta T}, \\
& R=\frac{4 \sigma^{*} T_{\infty}^{3}}{k^{*} k}, P r=\frac{v}{\alpha_{m}}, N b=\frac{\tau D_{B} \Delta \phi}{v}, N t=\frac{\tau D_{T} \Delta T}{v T_{\infty}}, S c=\frac{v}{\varepsilon D_{S}}, S r=\frac{\varepsilon D_{C T} \Delta T}{v \Delta C}, \\
& \beta=\frac{\varepsilon K_{0} \Delta C^{n-1}}{b}, L e=\frac{v}{\varepsilon D_{B}}, G_{1}=L\left(\frac{b}{v}\right)^{\frac{1}{2}}, G_{2}=K_{1}\left(\frac{b}{v}\right)^{\frac{1}{2}}, G_{3}=K_{2}\left(\frac{b}{v}\right)^{\frac{1}{2}}, G_{4}=K_{3}\left(\frac{b}{v}\right)^{\frac{1}{2}} .
\end{aligned}
$$

The physical quantities of interest are the skinfriction coefficient $C_{f}$, Nusselt number $N u x$, Sherwood number $S h x$ and Nanoparticle Sherwood $\tau_{w}=-\mu\left[\frac{\partial u}{\partial y}\right]_{y=0}=-\rho\left(v b^{3}\right)^{\frac{1}{2}} x f^{\prime \prime}(0)$. number $N n_{x}$. Further the shearing stress at the surface of the wall $\tau_{w}$ is given by

The skin friction coefficient is defined as

$C_{f}=\frac{\tau_{w}}{\rho u_{w}^{2}}$

and using equation(19) in the equation(20),we obtain

$C_{f} R e_{x}^{\frac{1}{2}}=-f^{\prime \prime}(0)$

The heat transfer rate at the surface flux at the wall is given by

$$
\begin{aligned}
q_{w} & =-k\left[\frac{\partial T}{\partial y}\right]_{y=0}-\frac{4 \sigma^{*}}{3 k^{*}}\left[\frac{\partial T^{4}}{\partial y}\right]_{y=0} \\
& =-k \Delta T\left(\frac{b}{v}\right)^{\frac{1}{2}}\left\{1+\frac{4}{3}\left(\frac{4 \sigma^{*} T_{\infty}^{3}}{k^{*} k}\right)\right\} \theta^{\prime}(0),
\end{aligned}
$$

The local Nusselt number is defined as

$N u_{x}=\frac{x q_{w}}{k \Delta T}$.

Using equation (22) in equation (23) the dimensionless reduced Nusselt number can be $N u_{r}=\frac{N u_{x}}{R e_{x}^{\frac{1}{2}}}=-\left\{1+\frac{4}{3} R\right\} \theta^{\prime}(0)$.

The mass flux at the surface of the wall is given by

$q_{m}=-D_{s}\left[\frac{\partial C}{\partial y}\right]_{y=0}=-D_{s} \Delta C\left(\frac{b}{v}\right)^{\frac{1}{2}} S^{\prime}(0)$.

The local Sherwood is defined as

$S h_{x}=\frac{x q_{m}}{D_{s} \Delta C}$.

Using (25) in (26) the dimensionless reduced Sherwood number obtained as

$S h_{r}=\frac{S h_{x}}{R e_{x}^{\frac{1}{2}}}=-S^{\prime}(0)$. 
The nanoparticle mass flux at the surface of the wall is defined as

$$
q_{n}=-D_{B}\left[\frac{\partial \phi}{\partial y}\right]_{y=0}=-D_{B} \Delta \phi\left(\frac{b}{v}\right)^{\frac{1}{2}} \gamma^{\prime}(0) .
$$

The local nanoparticle Sherwood number is defined as

$$
N n_{x}=\frac{x q_{n}}{D_{B} \Delta \phi} \text {. }
$$

Using (28) in (29) the dimensionless reduced nanoparticle Sherwood number is defined as

$$
N n_{r}=\frac{N n_{x}}{R e_{x}^{\frac{1}{2}}}=-\gamma^{\prime}(0) \text {. }
$$

where $R e_{x}=\frac{x u_{w}}{v}$ is the local Reynolds number.

\section{RESULTS AND DISCUSSION}

The set of ordinary differential equations (11)-(14) subject to the boundary conditions (15) (18) was solved numerically for various ranges of thermal convective, nanoparticle mass convective, Soret number, chemical reaction parameter, Lewis number, Brownian motion parameter, thermophoresis parameter and slip factors. Numerical results were obtained by using the MATLAB bvp4c solver. As the test of accuracy of the solution ,the values of compared with the values reported by Hayat et al. [23], Andersson [12] and Ibrahim\& Shankar [24] for different values of the slip factor in Table 1 . The result show that the numerical results obtained by the present routine are in very good agreement with previous results. With increasing velocity slip parameter, the skin friction coefficient reduces. Table 2 shows the values of the reduced Nusselt number for various values of Prandtl number in the absence of all other parameters. The results in Table 2 are compared with Grubka \& Bobba [3]. The comparison presented in Table 2 show a good agreement with results in the literature.

Table 1: Comparison of skin friction coefficient - $\mathrm{f}^{\prime \prime}(0)$ for different values of $G_{1}$ when for $\lambda_{1}=\lambda_{2}=N c=$ $P r=N t=N b=R=S c=S r=\beta=n=L e=G_{2}=G_{3}=0$ and $G_{4}=0$

\begin{tabular}{|c|c|c|c|c|}
\hline$G_{1}$ & $\begin{array}{c}\text { Present } \\
\text { Results }\end{array}$ & $\begin{array}{c}\text { Hayat et al. } \\
{[23]}\end{array}$ & $\begin{array}{c}\text { Andersso } \\
\mathrm{n}[12]\end{array}$ & $\begin{array}{c}\text { Ibrahim \& Shankar } \\
{[24]}\end{array}$ \\
\hline 0.0 & 1.000000 & 1.000000 & 1.0000 & 1.0000 \\
\hline 0.1 & 0.872082 & 0.872082 & 0.8721 & 0.8721 \\
\hline 0.2 & 0.776377 & 0.776377 & 0.7764 & 0.7764 \\
\hline 0.5 & 0.591195 & 0.591195 & 0.5912 & 0.5912 \\
\hline 2.0 & 0.283980 & 0.283981 & 0.2840 & 0.2840 \\
\hline 5.0 & 0.144840 & 0.144841 & 0.1448 & 0.1448 \\
\hline 10.0 & 0.081242 & 0.081249 & 0.0812 & 0.0812 \\
\hline 20.0 & 0.043788 & 0.043782 & 0.0438 & 0.0438 \\
\hline 50.0 & 0.018596 & 0.018634 & 0.0186 & 0.0186 \\
\hline
\end{tabular}

Table 2: Comparison of wall temperature gradient $-\theta^{\prime}(0)$ for different values of $\operatorname{Pr}$ when for $\lambda_{1}=\lambda_{2}=N c=$ $N t=N b=R=S c=S r=\beta=n=L e=G_{1}=G_{2}=G_{3}=0$ and $G_{4}=0$

\begin{tabular}{ccc}
\hline Pr & Present Results & Grubka \& Bobba[3] \\
\hline 0.01 & 0.029418 & 0.0294 \\
0.72 & 1.088524 & 1.0885 \\
1.0 & 1.333333 & 1.3333 \\
3.0 & 2.509725 & 2.5097 \\
10.0 & 4.796873 & 4.7969 \\
100.0 & 15.711967 & 15.7120 \\
\hline
\end{tabular}


Figure 2: Effects chemical reaction $\beta$ and order of the reaction $n$ on velocity (a) and (b) solute concentration for $\lambda_{1}=\lambda_{2}=1.2, N c=0.2, \operatorname{Pr}=7, N t=N b=0.3, R=2, S c=0.6, S r=0.5, L e=2, G_{1}=G_{2}=G_{3}=$

$$
G_{4}=0.5
$$

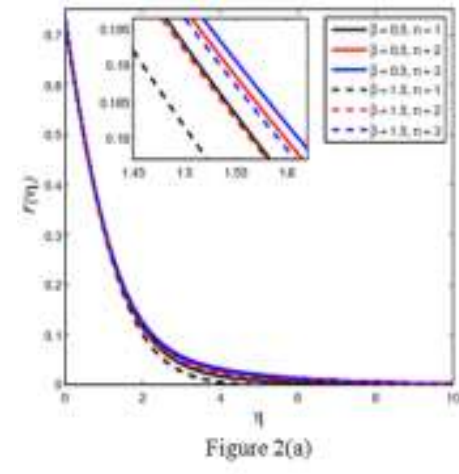

The effects of thermal convection, nanoparticle mass convection, double diffusive buoyancy, Brownian motion and thermophoresis, Schmidt number, Soret number, Chemical reaction, Lewis number, slip parameters are shown in Figures for various fluid properties. The velocity and solute concentration profiles for various values of chemical reaction parameter and various orders of chemical reaction $n$ are shown in Figure 2. It is interesting to note that the momentum boundary layer thickness increases by increasing the order of chemical reaction. It can also

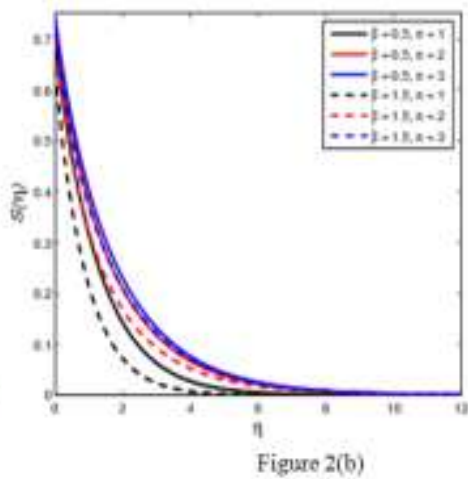

be noted that for a fixed order of chemical reaction, the velocity profile increases with the chemical reaction parameter. Figure 2(b) shows the variation of species concentration for different orders of the chemical reaction $n$ and chemical reaction parameter. The results show that the concentration profiles increase with an increase in the order of the chemical reaction. For higher order reactions, the rate of increase in the concentration is less compared with lower order readions

Figure 3: Effects of Schmidt number and chemical reaction on velocity (a) and (b) solute concentration for $\lambda_{1}=\lambda_{2}=1.2, N c=0.2, \operatorname{Pr}=7, N t=N b=0.3, R=2, S r=0.5, n=2, L e=2, G_{1}=G_{2}=G_{3}=G_{4}=0.5$

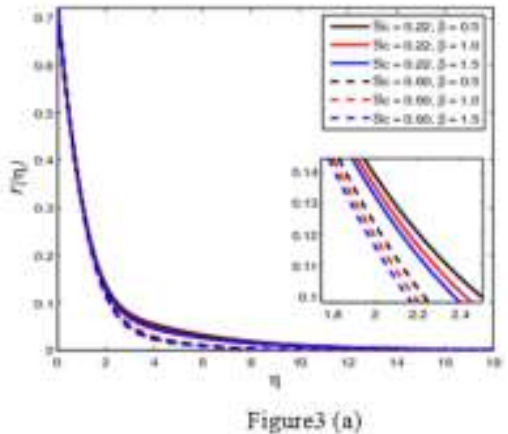

The influence of the Schmidt number and the chemical reaction on velocity and concentration profiles is shown in Figure 3. The Schmidt number signifies the relative influence of momentum diffusion to species diffusion. When $S c$ equals unity both the momentum and the species diffuse at the same rate. In the present analysis we have chosen the values of Schmidt number those represent the diffusing chemical species of common interest. The values of $\mathrm{Sc}$ for $\mathrm{H}_{2}$ and $\mathrm{H}_{2} \mathrm{O}$ are 0.22 and 0.6 respectively. As the schmidt number increases, the velocity and concentration boundary layer thicknesses decrease. This may be physically explained by the fact that molecular diffusivity decreases which reduces the ability of the chemical species to diffuse in the flow

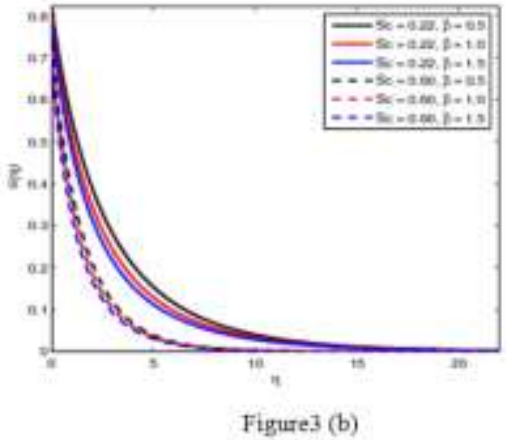

regime. We also note that for heavier diffusing species, the concentration boundary layer thickness reduces with an increase in chemical reaction parameter. In Figure 3 (b), the effect of increasing $\beta$ and $S c$ on the concentration profiles is shown. We note that an increase in the chemical reaction influences the concentration and therefore an increase in the chemical reaction parameter reduces the concentration boundary layer thickness of $\mathrm{H}_{2}$ and $\mathrm{H}_{2} \mathrm{O}$. However, in the case of heavier diffusing species the reduction in the concentration boundary layer thickness is less compared with the lighter diffusing particles. For large values of $\eta$ the effect dies out and so the concentration boundary layer thickness remains almost unchanged. Figures 4 and 5 
demonstrate the effects of various parameters on skin friction, heat transfer, mass transfer and nanoparticle mass transfer profiles. From figure 4 we note that the skin friction coefficient increases with an increase in the order of the chemical reaction for fixed values of $\lambda_{1}, \lambda_{2}$. We also observe that with an increase in $\lambda_{1}$ and $\lambda_{2}$ the skin friction coefficient decreases as the order of the reaction decreases. It is worth noting that the heat transfer rate decreases with an increase in the order of the chemical reaction and that for higher order reactions the rate of increase in heat transfer coefficient is less compared to the rate of increase for lower order reactions. More over, the heat transfer rate increases with an increase in the values of $\lambda_{1}$ and $\lambda_{2}$. The influence of $\lambda_{1}$ and $\lambda_{2}$ on the Sherwood number is less significant compared to that on the Nusselt number. From Figure 5 (a) we observe that the surface mass transfer rate increases quite rapidly with an increase in $\beta$ and decreases with an increase in $n$. These results are similar to the results of Rahman and Al-Lawatia [20]. Figure 5 (b) shows that the nanoparticle mass transfer rate increases with an increase in the order of the chemical reaction. For lower values of $\lambda_{1}$ and $\lambda_{2}$ the change in the nanoparticle Sherwood number is less compared with that at higher values.

Figure 4: (a) Skin friction and (b) Heat transfer as functions of the chemical reaction parameter $\beta$, when $N c=0.2, \operatorname{Pr}=10, N t=N b=0.3, R=2, S c=S r=0.5, L e=2, G_{1}=G_{2}=G_{3}=G_{4}=0.5$
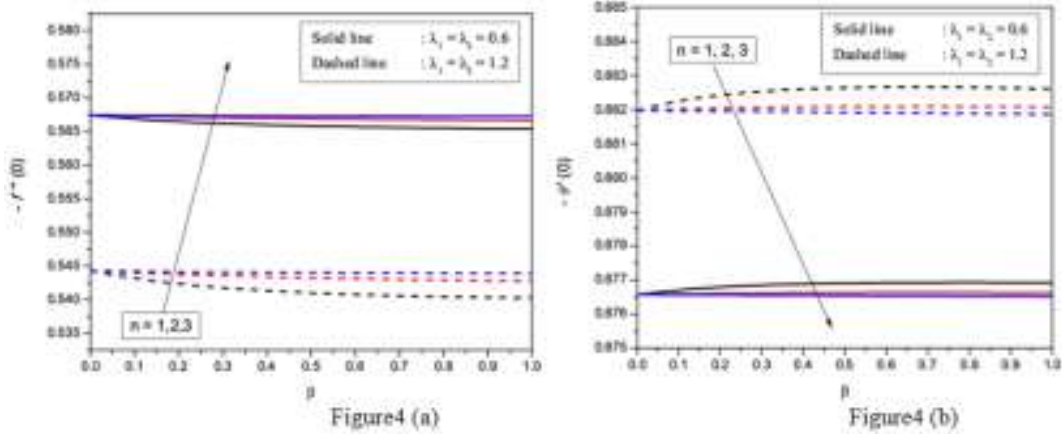

Figure 5: (a) Mass transfer and (b) nanoparticle mass transfer as functions of the chemical reaction parameter $\beta$, when $N c=0.2, P r=10, N t=N b=0.3, R=2, S c=S r=0.5, L e=2, G_{1}=G_{2}=G_{3}=G_{4}=0.5$
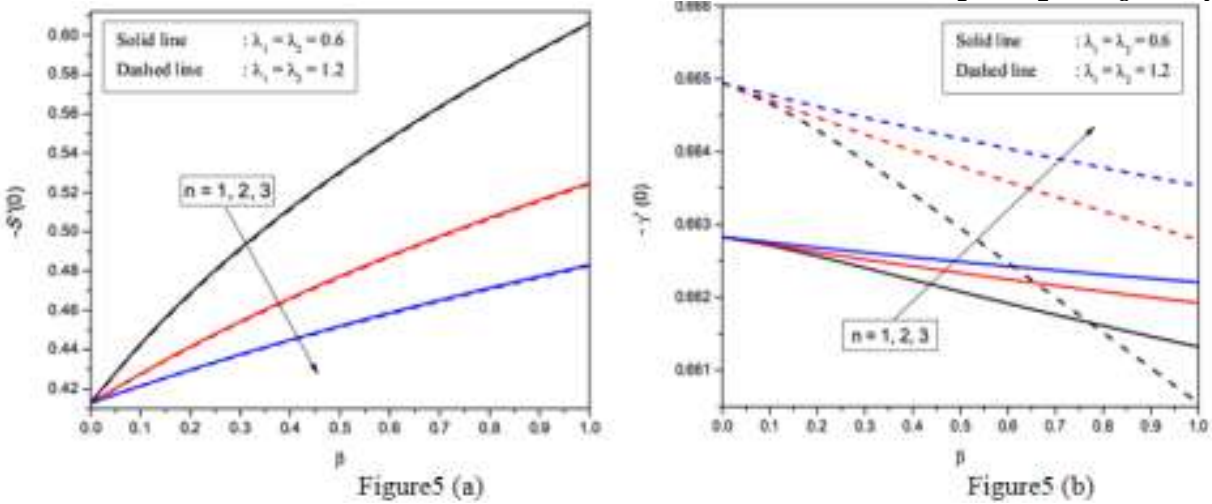

Figure 6: (a) Skin friction and (b) mass transfer as functions of the chemical reaction parameter $\beta$, when $\lambda_{1}=\lambda_{2}=0.8, N c=0.3, \operatorname{Pr}=10, R=2, S c=S r=0.5, L e=2, G_{1}=G_{2}=G_{3}=G_{4}=0.5$
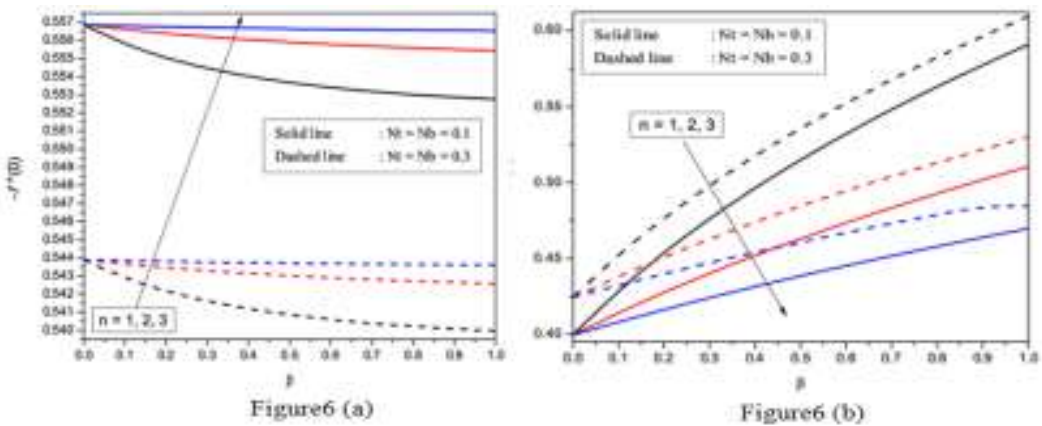
Figure 7: Effect of nanoparticle mass transfer as a function of chemical reaction parameter $\beta$, when $\lambda_{1}=\lambda_{2}=$ $0.8, N c=0.3, \operatorname{Pr}=10, R=2, S c=S r=0.5, L e=2, G_{1}=G_{2}=G_{3}=G_{4}=0.5$

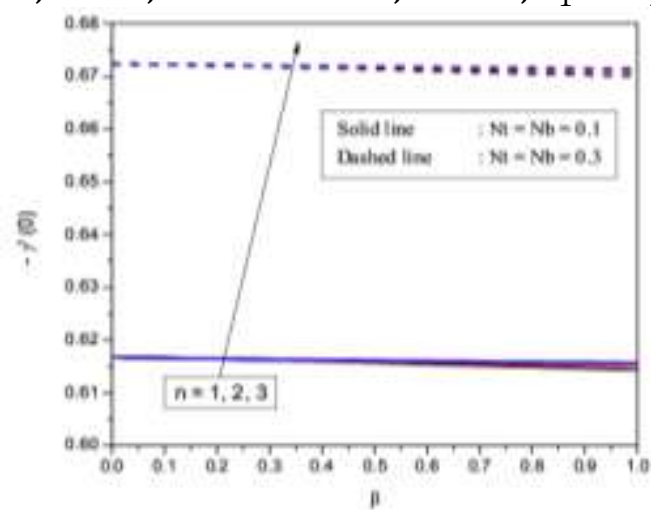

Figure7

Figure 8: (a) Effects of skin friction and (b) heat transfer as a function of chemical reaction parameter $\beta$, when $\lambda_{1}=\lambda_{2}=0.6, N c=0.2, P r=10, N t=N b=0.3, R=2, S r=0.5, L e=2, G_{1}=G_{2}=G_{3}=$

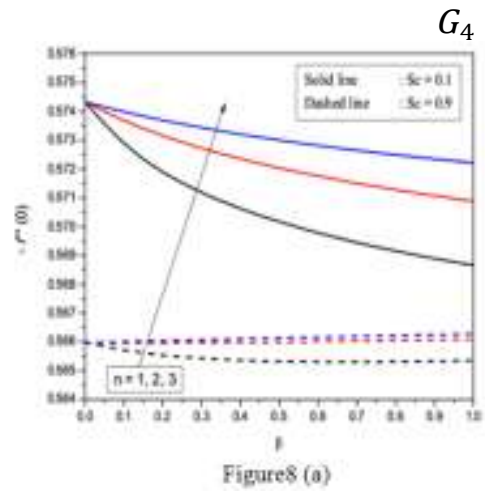
$G_{4}=0.5$

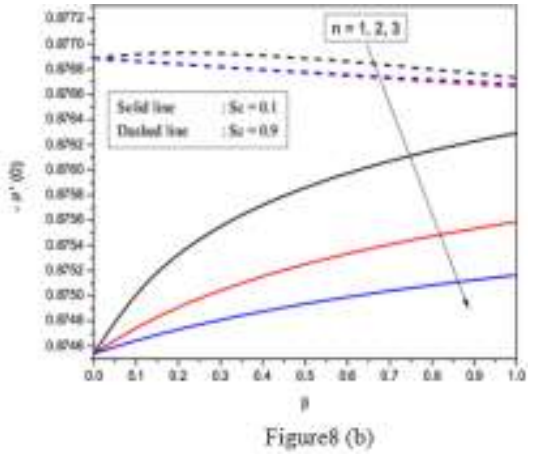

Figure 9: Effects of the chemical reaction parameter $\beta$ on mass transfer when $\lambda_{1}=\lambda_{2}=0.6, N c=$ $0.2, P r=10, N t=N b=0.3, R=2, S r=0.5, L e=2, G_{1}=G_{2}=G_{3}=G_{4}=0.5$

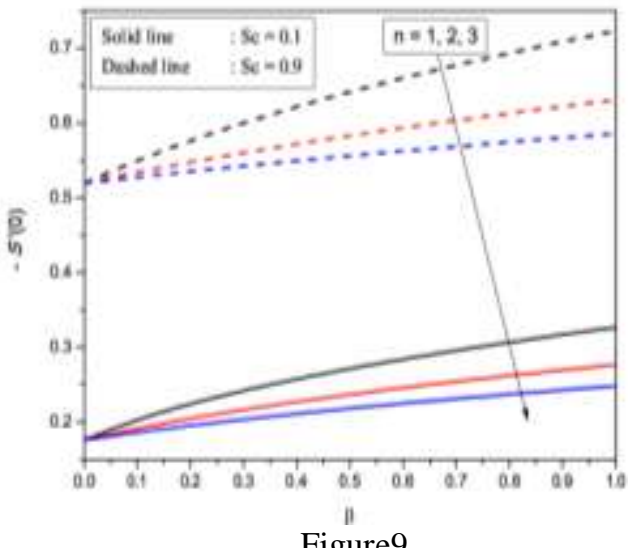

Figure9

The skin friction, heat transfer, mass transfer and nanoparticle mass transfer values are plotted in Figures $8-9$ in relation to the chemical reaction parameter $\beta$. The skin friction coefficient is seen to increase for decreasing values of $S c$. This behaviour is due to the fact that an increase in the Schmidt number leads to thicker velocity profiles which in turn give lower skin friction values. As the chemical reaction parameter increases higher skin friction values are obtained for third order chemical reactions. For smaller values of the Schmidt number the dispersion in the flow profiles are found to be significant. In the presence of heavier species (large $S c$ ) the heat transfer rate decrease is more significant compared to the lighter species. As shown in Figure 9, the Schmidt number plays an important role in determination of mass transfer. A rise in the Schmidt number leads to an increase in the mass transfer rate. 
With increasing order of chemical reaction, the nanoparticle Sherwood number is found to increase for $S c=0.1$. The opposite is true in the case when $S c$ $=0.9$.

\section{CONCLUSIONS}

In this study we have investigated higher order chemical reaction effects on heat and mass transfer over a permeable stretching sheet with slip boundary conditions. The governing differential equations were solved numerically using the bvp4c solver in Matlab. The following conclusions may be drawn from this study

1. For higher order chemical reactions, the rate of increase in the concentration profiles is less significant compared with the lower order reactions.

2. As increase in the Schmidt number leads to a reduction in the velocity and concentration boundary layer thicknesses.

3. An increase in the velocity slip parameter enhances the nanoparticle concentration.

4. With an increase in $\lambda_{1}$ and $\lambda_{2}$, the skin friction coefficient decreases, and the reduction is more significant for higher order reactions compared to lower order chemical reactions.

5. The Sherwood number increases with decreases in the Lewis number.

\section{REFERENCES}

[1] Crane, L.J., Flow past a stretching plate, Z Angew. Math. Phys. (ZAMP) 21, 645 - 647 (1970).

[2] Erickson, L.E., L.T. Fan and V.G. Fox, Heat and mass transfer on a moving continuous flat plate with suction or injection, Ind. Eng. Chem. Fundam. 5, 19 - 25 (1966).

[3] Grubka, L.J. and K.M. Bobba, Heat transfer characteristics of a continuous, stretching surface with variable temperature, ASME J. Heat Transfer 107, 248 - 250 (1985).

[4] Chen, C.-H., "Laminar mixed convection adjacent to vertical, continuously stretching sheets", Heat Mass Transfer 33, 471 - 476 (1998).

[5] Abo-Eldahab, E.M. and M.A. El-Aziz, Blowing/suction effect on hydromagnetic heat transfer by mixed convection from an inclined continuously stretching surface with internal heat generation/absorption, Int. J. Therm. Sci. 43, 709 - 719 (2004).

[6] Lauga. E., M.P. Brenner and H. A. Stone, Handbook of experimental fluid dynamics, edited by C. Tropea, A. Yarin, J. Foss, Springer, New York, Chap. 19, 1219 - 1240 (2007).

[7] Neto, C., D.R. Evans, E. Bonaccurso, H.J. Butt, and V.S.J. Craig, Boundary slip in
Newtonian liquids: a review of experimental studies, Rep. Prog. Phys. 68, 2859 - 2897 (2005).

[8] Bocquet. L and J.-L. Barrat, Flow boundary conditions from nano-to micro-scales, Soft Matter 3, 685- 693 (2007).

[9] Gad-el-Hak, M., The fluid mechanics of microdevices-The freeman scholar Lecture, J. Fluids Eng. 121, 5 - 33 (1999).

[10] Arlemark, E.J, S.K. Dadzie and J.M. Reese, An extension to the Navier-Stokes equations by considering molecular collisions with boundaries, J. Heat Transfer 132, 041006 (2010).

[11] Wang. C.Y.,Flow due to a stretching boundary with partial slip-an exact solution of the Navier-Stokes equations, Chem. Eng. Sci. 57, 3745 - 3747 (2002).

[12] Andersson, H.I., Slip flow past a stretching surface, Acta Mechanica, 158, 121 - 125 (2002).

[13] Bhattacharyya, K., S. Mukhopadhyay and G.C. Layek, Slip effects on boundary layer stagnation-point flow and heat transfer towards a shrinking sheet, Int. J. Heat Mass transfer 54, 308 - 313 (2011).

[14] Fang. T., S. Yao, J. Zhang and A. Aziz, Viscous flow over a shrinking sheet with a second order slip flow model, Commun. Nonlinear Sci. Numer. Simul. 15, 1831 1842 (2010).

[15] Bhattacharyya, K. and G.C. Layek, Similarity solution of MHD boundary layer flow with diffusion and chemical reaction over a porous flat plate with suction/blowing, Meccanica 47, 1043 - 1048 (2012).

[16] Hossain, M.A. and H.S. Takhar, Radiation effect on mixed convection along a vertical plate with uniform surface temperature, Heat Mass Transfer 31, 243 - 248 (1996).

[17] Takhar,H.S., R.S.R. Gorla and V.M. Soundalgekar, Radiation effects on MHD free convection flow of a gas past a semiinfinite vertical plate, Int. J. Numer. Meth. Heat Fluid Flow 6, 77 - 83 (1996).

[18] Hossain, M.A., M.A. Alim and D.A.S. Rees, The effect of radiation on free convection from a porous vertical plate, Int. J. Heat Mass Transfer 42, 181 - 191 (1999).

[19] Mukhopadhyay, S. and R.S.R. Gorla, Effects of partial slip on boundary layer flow past a permeable exponential stretching sheet in presence of thermal radiation, Heat Mass Transfer 48, 1773 - 1781 (2012).

[20] Rahman, M.M., and M. Al-Lawatia, Effects of higher order chemical reaction on micropolar fluid flow on a power law permeable stretched sheet with variable 
concentration in a porous medium, Can. J.

Chem. Eng. 88, 23 - 32(2010).

[21] Andersson, H.I., O.R. Hansen and B. Holmedal, Diffusion of a chemically reactive species from a stretch- ing sheet, Int.J. Heat Mass transfer 37, 659 - 664 (1994).

[22] Takhar, H.S., A.J. Chamkha and G. Nath, Flow and mass transfer on a stretching sheet with a magnetic field and chemically reactive species, Int. J. Eng. Sci. 38, 1303 1314 (2000).

[23] Hayat, T., M. Qasim and S. Mesloub, MHD flow and heat transfer over permeable stretching sheet with slip conditions. Int. J. Numer. Meth. Fluids 66, 963 - 975 (2011).

[24] Ibrahim. W. and B. Shankar, MHD boundary layer flow and heat transfer of a nanofluid past a permeable stretching sheet with velocity, thermal and solutal slip boundary conditions, Computers \& Fluids 75, 1- 10 (2013).

[25] Sharma. R., A. Ishak and I. Pop, Partial slip flow and heat transfer over a stretching sheet in a nanofluid, Mathematical Problems in Engineering, (2013) Article ID 724547, 7 pages.

[26] Modest. M.F., Radiation Heat Transfer, McGraw-Hill, USA (1993) (Chapter 13) 